\title{
Density Elimination and Rational Completeness for First-Order Logics ${ }^{\star}$
}

\author{
Agata Ciabattoni ${ }^{1}$ and George Metcalfe ${ }^{2}$ \\ 1 Institute of Discrete Mathematics and Geometry, Technical University Vienna, \\ Wiedner Hauptstrasse 8-10, A-1040 Wien, Austria. agata@logic . at \\ 2 Department of Mathematics, Vanderbilt University \\ 1326 Stevenson Center, Nashville TN 37240, USA. \\ george.metcalfeevanderbilt.edu
}

\begin{abstract}
Density elimination by substitutions is introduced as a uniform method for removing applications of the Takeuti-Titani density rule from proofs in firstorder hypersequent calculi. For a large class of calculi, density elimination by this method is guaranteed by known sufficient conditions for cut-elimination. Moreover, adding the density rule to any axiomatic extension of a simple first-order logic gives a logic that is rational complete; i.e., complete with respect to linearly and densely ordered algebras: a precursor to showing that it is a fuzzy logic (complete for algebras with a real unit interval lattice reduct). Hence the sufficient conditions for cut-elimination guarantee rational completeness for a large class of first-order substructural logics.
\end{abstract}

\section{Introduction}

Elimination of the cut-rule is a fundamental topic in proof theory, corresponding to the removal of lemmas in proofs. However, the addition and subsequent elimination of other rules can also be of considerable interest. In this paper we consider one such rule of importance in Fuzzy Logic: the so-called "density rule" of Takeuti and Titani [14]:

$$
\frac{T \vdash(A \rightarrow p) \vee(p \rightarrow B) \vee C}{T \vdash(A \rightarrow B) \vee C} \text { (density) }
$$

where $p$ is a propositional variable not occurring in $T, A, B$, or $C$. Ignoring $T$ and $C$, the negation of the conclusion may (roughly) be interpreted as " $A>B$ " and the negation of the premise as "for some $p: A>p$ and $p>B$ ". That is, between every two elements there exists another element. Note that adding this rule to any axiomatization of Classical Logic leads to inconsistency (e.g. take $A$ to be $\top$ and $B$ to be $\perp$ ).

The density rule was used by Takeuti and Titani to axiomatize Intuitionistic Fuzzy Logic [14], better known as first-order Gödel logic, one of the main formalizations of Fuzzy Logic [8]. Alternative axiomatizations by Horn and Takano [13] show that (density) is not required; giving a kind of "semantic elimination" of the rule. Baaz and Zach [2] have also provided a syntactic elimination of (density) using a proof

\footnotetext{
* Work Supported by FWF Project P18731.
} 
system for first-order Gödel logic in the framework of hypersequents, a generalization of Gentzen sequents introduced by Avron [1]. This elimination procedure follows the spirit of Gentzen's cut-elimination method, proceeding by induction on the height of a proof of the premise and shifting applications of the rule upwards.

In [10], Metcalfe and Montagna recognized that these two steps - adding and eliminating the density rule - provide a general method for establishing the so-called "standard completeness" of $t$-norm ${ }^{3}$ based (and related) fuzzy logics: that is, completeness of axiomatic systems with respect to algebras whose lattice reduct is the real unit interval $[0,1]$. In particular, it was shown that any axiomatic extension of the elementary propositional fuzzy logic UL extended with (density) is complete with respect to corresponding linearly and densely ordered algebras. This constitutes the so-called "rational completeness" step of the proof. Standard completeness may then be obtained in many cases (but not in general) by means of the Dedekind-MacNeille completion. It was also shown in [10] that for particular propositional logics possessing a suitable hypersequent calculus, density elimination can be established (Gentzen-style, following [2]) thereby giving standard completeness for the original logic without density. This general approach is in contrast to more logic-specific "semantic" techniques for proving standard completeness $[8,9,6,11]$.

The contribution of this paper is in two parts. First, we introduce a new general method for density elimination. In this approach, similar to normalization for natural deduction systems, applications of the density rule are removed by making suitable substitutions for the new propositional variables. This avoids the combinatorial difficulties of the Gentzen-style proofs in [2,10]. Applying this method to first-order single-conclusion hypersequent calculi with weakening rules, we are able to show that the syntactic conditions defined in [3] for cut-elimination also guarantee density elimination. In the second part of the paper we show that adding the density rule to any axiomatic extension of the first-order version of $\mathbf{U L}$, gives a logic that is complete with respect to linearly and densely ordered algebras. Combining the two parts we obtain rational completeness for a wide class of first-order fuzzy logics with weakening (see [5] for details). These include the first-order versions of (the logic of left-continuous $t$-norms) Monoidal $t$-norm based logic MTL [7] (proved standard complete in [11] by a different method) and its extensions SMTL [6] and CnMTL (with $n \geq 2$ ) [4].

\section{2 (Hyper)Sequent Calculi}

We consider formulae built over a vocabulary $\mathcal{V}$ consisting of (countably many): (term) variables $x, y, z, \ldots$, for each $n \geq 0$, constants $c, d, \ldots, n$-ary predicate symbols, as well as $m$-ary connectives $\star_{1}, \star_{2}, \ldots$ for each $m \geq 0$ and the quantifiers $\forall$ and $\exists$. Terms are defined in the usual way. A formula (in the vocabulary $\mathcal{V}$ ) is either an atomic formula or a compound formula of the form $\star_{i}(\boldsymbol{A})$ or $\mathcal{Q} x A$ with $\star_{i}$ an $m$-ary connective, which connect formulae $\boldsymbol{A} \equiv A_{1}, \ldots, A_{n}$ and $\mathcal{Q} \in\{\forall, \exists\}$. For convenience we call nullary predicate symbols, propositional variables, denoted by $p, q, \ldots$.

We indicate with $\Gamma, \Delta, \Pi, \Sigma, \ldots$ (possibly empty) multisets of formulae. To specify inference rules we use meta-variables $X, Y, Z$ standing for arbitrary formulae and

\footnotetext{
${ }^{3} t$-norms are the main tool in Fuzzy Logic for conjunctively combining vague information.
} 
$\Theta, \Xi, \Phi, \Psi, \Upsilon, \ldots$ standing for (possibly empty) multisets of meta-variables. When $\lambda \geq$ $0, \Gamma^{\lambda}$ (resp. $\Theta^{\lambda}$ ) denotes $\Gamma, \ldots, \Gamma$ (resp. $\Theta, \ldots, \Theta$ ), $\lambda$ times. A (meta) sequent $\Gamma \Rightarrow \Delta$ $(\Theta \Rightarrow \Xi)$, where $\Gamma(\Theta)$ is said to be antecedent and $\Delta(\Xi)$ consequent, is singleconclusion if $\Delta(\Xi)$ contains at most one formula (meta-variable). A sequent calculus is single-conclusion if all its sequents are single-conclusion.

Definition 1. We call any propositional single-conclusion sequent calculus $\mathbf{L}_{\mathbf{L}}$ simple whenever $\mathbf{L}_{\mathbf{L}}$ consists of the identity axiom of the form $X \Rightarrow X$, together with: the (multiplicative version of the) cut rule $(C U T)$, structural rules $\left\{\left(r_{i}\right)\right\}_{i \in \Lambda_{0}}$ and for each logical connective $\star$, left logical rules $\left\{(\star, l)_{j}\right\}_{j \in \Lambda_{1}}$ and right logical rules $\left\{(\star, r)_{k}\right\}_{k \in \Lambda_{2}}\left(\Lambda_{0}, \Lambda_{1}, \Lambda_{2}\right.$ can be empty):

$$
\begin{array}{ll}
\frac{\Theta \Rightarrow X \quad \Theta^{\prime}, X \Rightarrow \Xi}{\Theta, \Theta^{\prime} \Rightarrow \Xi}(C U T) & \frac{\Upsilon_{1} \Rightarrow \Psi_{1} \cdots \Upsilon_{n} \Rightarrow \Psi_{n}}{\Theta \Rightarrow \Xi}\left(r_{i}\right) \\
\frac{\Upsilon_{1} \Rightarrow \Psi_{1} \quad \cdots \Upsilon_{n} \Rightarrow \Psi_{n}}{\Theta, \star(\boldsymbol{X}) \Rightarrow \Xi}(\star, l)_{j} & \frac{\Upsilon_{1} \Rightarrow \Psi_{1} \cdots \Upsilon_{n} \Rightarrow \Psi_{n}}{\Theta \Rightarrow \star(\boldsymbol{X})}(\star, r)_{k}
\end{array}
$$

In the rules $\left(r_{i}\right),(\star, l)_{j}$, and $(\star, r)_{k}, n \geq 0$ and the meta-variables in $\Theta$ (called left context meta-variables), those in $\Xi$ (called right context meta-variables), and the metavariables in $\boldsymbol{X} \equiv X_{1}, \ldots, X_{m}, m \geq 0$ (called active meta-variables) are mutually disjoint. In addition, the structural (and logical) rules satisfy the following condition: $(*)$ Any meta-variable in $\Upsilon_{1}, \ldots, \Upsilon_{n}$ is a left context meta-variable (or an active metavariable), and any meta-variable in $\Psi_{1}, \ldots, \Psi_{n}$ is a right context meta-variable (or an active meta-variable).

As usual, an instance of a logical or structural rule is obtained by substituting arbitrary formulae for meta-variables. In an instance of a logical or structural rule, the formulae replacing context meta-variables (active meta-variables, respectively) are called context formulae (active formulae, respectively) and formulae of the form $\star(\boldsymbol{A})$ as well as the formulae replacing $X$ in identity axioms are called principal formulae. The two occurrences of the formula instantiating $X$ in $(C U T)$ are called cut formulae. Proofs (or derivations) are defined in the usual way.

Definition 2. A w-simple calculus is a simple sequent calculus containing the weakening rules $(w, l)$ and $(w, r)$ of Fig. 1 . A first-order (w-)simple sequent calculus is a (w-)simple sequent calculus extended with the rules for quantifiers in Gentzen's calculus $\mathbf{L} \mathbf{J}$ for intuitionistic logic.

Hypersequent calculi arise by extending Gentzen calculi to refer to many (a multiset of) sequents, instead of just one. Introduced by Avron in [1], they are particularly suitable for dealing with logics with the linearity axiom $(\operatorname{lin})(A \rightarrow B) \vee(B \rightarrow A)$, prominent examples being $t$-norm based fuzzy logics [8].

Definition 3. A hypersequent is a multiset $S_{1}|\ldots| S_{n}$ where each $S_{i}$ for $i=1 \ldots n$ is a sequent, called a component of the hypersequent. A hypersequent is called singleconclusion if all its components are single-conclusion.

We will assume from now on that we deal only with single-conclusion (hyper)sequent calculi. Like sequent calculi, hypersequent calculi consist of initial hypersequents, logical rules, and structural rules, where we write rules using meta-sequents and a variable 


\begin{tabular}{|c|c|}
\hline$\overline{X \Rightarrow X}^{(I D)} \quad \overline{\Theta, \perp \Rightarrow \Xi}^{(\perp)}$ & $\frac{\Theta \Rightarrow X \quad \Phi, X \Rightarrow \Xi}{\Theta, \Phi \Rightarrow \Xi}(C U T)$ \\
\hline$\frac{\Theta \Rightarrow \Xi}{\Theta, X \Rightarrow \Xi}(w, l)$ & $\frac{\Theta \Rightarrow}{\Theta \Rightarrow X}(w, r)$ \\
\hline$\frac{\Theta, X, Y \Rightarrow \Xi}{\Theta, X \odot Y \Rightarrow \Xi}(\odot, l)$ & $\frac{\Theta \Rightarrow X \quad \Theta^{\prime} \Rightarrow Y}{\Theta, \Theta^{\prime} \Rightarrow X \odot Y}(\odot, r)$ \\
\hline$\frac{\Theta, X_{i} \Rightarrow \Xi}{\Theta, X_{1} \odot X_{2} \Rightarrow \Xi}(\wedge, l)_{i=1,2}$ & $\frac{\Theta \Rightarrow X \quad \Theta \Rightarrow Y}{\Theta \Rightarrow X \wedge Y}(\wedge, r)$ \\
\hline$\frac{\Theta, Y \Rightarrow \Xi \quad \Theta^{\prime} \Rightarrow X}{\Theta, \Theta^{\prime}, X \rightarrow Y \Rightarrow \Xi}(\rightarrow, l)$ & $\frac{\Theta, X \Rightarrow Y}{\Theta \Rightarrow X \rightarrow Y}(\rightarrow, r)$ \\
\hline$\frac{\Theta, X \Rightarrow \Xi \quad \Theta, Y \Rightarrow \Xi}{\Theta, X \vee Y \Rightarrow \Xi}(\vee, l)$ & $\frac{\Theta \Rightarrow X_{i}}{\Theta \Rightarrow X_{1} \vee X_{2}}(\vee, r)_{i=1,2}$ \\
\hline
\end{tabular}

Fig. 1: The sequent calculus $\mathbf{F L}_{\text {ew }}$.

$\mathcal{G}$ (with an instance $G$ ) standing for an arbitrary hypersequent. Logical rules for connectives are then the same as those in sequent calculi, except that a "side hypersequent" may occur, denoted by $\mathcal{G}$. Structural rules are divided into two categories. Internal rules deal with formulae within sequents as in sequent calculi. External rules manipulate whole sequents. For example, external weakening and contraction rules $(E W)$ and $(E C)$ add and contract components respectively:

$$
\frac{\mathcal{G}}{\mathcal{G} \mid \Theta \Rightarrow \Xi}(E W) \quad \frac{\mathcal{G}|\Theta \Rightarrow \Xi| \Theta \Rightarrow \Xi}{\mathcal{G} \mid \Theta \Rightarrow \Xi}(E C)
$$

while the key rule to deal with the axiom $(\operatorname{lin})$ is Avron's communication rule $(C O M)$ which permits interaction between sequents:

$$
\frac{\mathcal{G}\left|\Theta, \Theta^{\prime} \Rightarrow \Xi \mathcal{G}\right| \Theta_{1}, \Theta_{1}^{\prime} \Rightarrow \Xi^{\prime}}{\mathcal{G}\left|\Theta, \Theta_{1} \Rightarrow \Xi\right| \Theta^{\prime}, \Theta_{1}^{\prime} \Rightarrow \Xi^{\prime}}(C O M)
$$

For a sequent rule with premises $S_{1} \ldots S_{n}$ and conclusion $S$, its hypersequent version is the rule with premises $\mathcal{G}\left|S_{1} \ldots \mathcal{G}\right| S_{n}$ and conclusion $\mathcal{G} \mid S$. E.g., the hypersequent version of the quantifier rules $(\exists, l)$ and $(\forall, r)$ are:

$$
\frac{\mathcal{G} \mid \Theta \Rightarrow Y(a)}{\mathcal{G} \mid \Theta \Rightarrow(\forall x) Y(x)}(\forall, r) \quad \frac{\mathcal{G} \mid Y(a), \Theta \Rightarrow \Xi}{\mathcal{G} \mid(\exists x) Y(x), \Theta \Rightarrow \Xi}(\exists, l)
$$

where the eigenvariable condition (on the rules' instances) applies to the whole hypersequent conclusions of the rules.

Definition 4. Let $\mathbf{L}_{\mathbf{L}}$ be any (first-order) w-simple sequent calculus. $\mathbf{H L}_{\mathbf{L}}^{\mathbf{C}}$, the hypersequent version of $\mathbf{L}_{\mathbf{L}}$ extended with $(C O M)$, consists of the hypersequent versions of the axioms and rules of $\mathbf{L}_{\mathbf{L}}$ plus $(E C),(E W)$, and $(C O M)$. 
Example 1. Let $\forall \mathbf{F} \mathbf{L}_{\text {ew }}$ be the first-order multiset version of the Full Lambek calculus with exchange and weakening [12] (see Fig. 1), roughly speaking a calculus for firstorder intuitionistic logic without contraction with an internalized exchange rule. We illustrate the use of $(C O M)$ with a proof of $(\operatorname{lin})$ in $\mathbf{H} \forall \mathbf{F L}_{\mathbf{e w}}^{\mathbf{C}}$, a hypersequent calculus for the first-order version of the logic of left-continuous $t$-norms MTL [7, 11]:

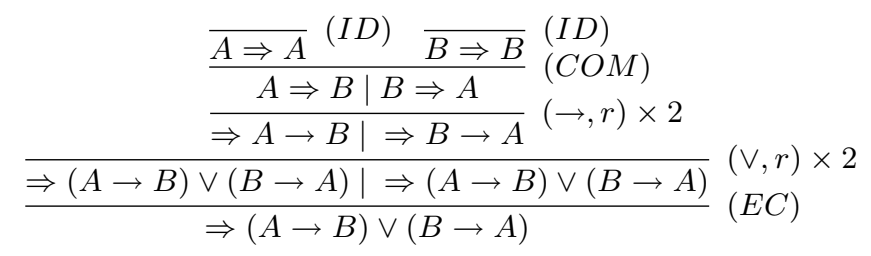

\section{Criteria for Cut-Elimination}

Syntactic criteria for the preservation of cut-elimination when a sequent calculus $\mathbf{L}_{\mathbf{L}}$ is "lifted" to the hypersequent calculus $\mathbf{H L}_{\mathbf{L}}^{\mathbf{C}}$ were introduced in [3]. The criteria intuitively say that (a) any application of $(C U T)$ can be shifted upwards over each premise's rule (i.e. rules are substitutive) and (b) each $(C U T)$ in which the cut formula is principal in both premises can be replaced by applications of $(C U T)$ over smaller cut-formulae (i.e. logical rules are reductive).

Definition 5. Let $\mathbf{L}_{\mathbf{L}}$ be a simple sequent calculus. We call its logical rules $\left\{(\star, r)_{k}\right\}_{k \in \Lambda}$ and $\left\{(\star, l)_{l}\right\}_{l \in \Lambda^{\prime}}$ for introducing a logical connective $\star$ reductive in $\mathbf{L}_{\mathbf{L}}$ if either $\Lambda$ or $\Lambda^{\prime}$ is empty, or for any $k \in \Lambda, l \in \Lambda^{\prime}$, and instances :

$$
\frac{\Gamma_{1} \Rightarrow \Delta_{1} \quad \cdots \quad \Gamma_{n} \Rightarrow \Delta_{n}}{\Sigma \Rightarrow \star\left(A_{1}, \ldots, A_{p}\right)}(\star, r)_{k} \quad \frac{\Gamma_{1}^{\prime} \Rightarrow \Delta_{1}^{\prime} \quad \cdots \quad \Gamma_{m}^{\prime} \Rightarrow \Delta_{m}^{\prime}}{\Sigma^{\prime}, \star\left(A_{1}, \ldots, A_{p}\right) \Rightarrow \Pi}(\star, l)_{l}
$$

the sequent $\Sigma, \Sigma^{\prime} \Rightarrow \Pi$ is derivable from $\left\{\Gamma_{i} \Rightarrow \Delta_{i}\right\}_{1 \leq i \leq n}$ and $\left\{\Gamma_{i}^{\prime} \Rightarrow \Delta_{i}^{\prime}\right\}_{1 \leq i \leq m}$ using only $(C U T)$ with cut formulae in $\left\{A_{1}, \ldots, A_{p}\right\}$ and the structural rules of $\mathbf{L}_{\mathbf{L}}$.

Example 2. The logical rules for the connectives $\odot$ (multiplicative "and"), $\wedge$ (additive "and"), $\vee$, and $\rightarrow$ in the calculus $\mathbf{F L}_{e w}$ (see Fig. 1) are reductive.

Let $S$ be a sequent and $A$ a formula; we define:

$$
\begin{aligned}
{\left[S \hookleftarrow_{A}^{r}(\Sigma, A \Rightarrow \Pi)\right] } & =\{\Gamma, \Sigma \Rightarrow \Pi \mid S \equiv \Gamma \Rightarrow A\} \\
{\left[S \hookleftarrow_{A}^{l}(\Sigma \Rightarrow A)\right] } & =\left\{\Gamma, \Sigma^{\lambda} \Rightarrow \Delta \mid S \equiv \Gamma, A^{\lambda} \Rightarrow \Delta\right\}
\end{aligned}
$$

Definition 6. Let $\mathbf{L}_{\mathbf{L}}$ be a (first-order) simple sequent calculus. A rule $(r)$ is said to be substitutive in $\mathbf{L}_{\mathbf{L}}$ if for each instance of $(r)$ with premises $S_{1}, \ldots, S_{n}$ and conclusion $S_{0}$ the following condition holds:

(*) for any $c \in\{r, l\}$, context formula $A$ (right or left context formula, depending on c) and single-conclusion sequent $S^{\prime}$ (which does not contain any eigenvariable of $(r)$ ), every $U \in\left[S_{0} \hookleftarrow_{A}^{c} S^{\prime}\right]$ has a derivation from $\left[S_{1} \hookleftarrow_{A}^{c} S^{\prime}\right] \ldots\left[\begin{array}{lll}S_{n} \hookleftarrow_{A}^{c} & S^{\prime}\end{array}\right]$ using only the structural rules of $\mathbf{L}_{\mathbf{L}}$ and $(r)$. 
Example 3. All the rules of the calculus $\forall \mathbf{F} \mathbf{L}_{\text {ew }}$ (see Fig. 1 for $\mathbf{F L}_{\mathbf{e w}}$ ) are substitutive in $\forall \mathbf{F} \mathbf{L}_{\text {ew }}$, as are, e.g. the following forms of weak contraction $(n \geq 2)$ :

$$
\frac{\Theta, X, X \Rightarrow}{\Theta, X \Rightarrow}(w c) \quad \frac{\Theta, \Psi_{1}^{n} \Rightarrow \Xi \ldots, \Theta, \Psi_{n-1}^{n} \Rightarrow \Xi}{\Theta, \Psi_{1}, \ldots, \Psi_{n-1} \Rightarrow \Xi}(n c)
$$

Theorem 1 ([3]). Let $\mathbf{L}_{\mathbf{L}}$ be a (first-order) simple calculus in which (a) logical rules are reductive and (b) rules are substitutive, then $\mathbf{H L}_{\mathbf{L}}^{\mathbf{C}}$ admits cut-elimination.

\section{Density Elimination by Substitutions}

Instances of the density rule for hypersequent calculi are of the form:

$$
\frac{G|\Sigma, p \Rightarrow \Delta| \Gamma \Rightarrow p}{G \mid \Sigma, \Gamma \Rightarrow \Delta}(D)
$$

where $p$ is a propositional variable not occurring in $\Sigma, \Gamma, \Delta$, or $G$. We show here that the conditions given above for cut elimination also guarantee density elimination for the hypersequent version of any w-simple sequent calculus extended with $(C O M)$ and $(D)$. We introduce for this purpose density elimination by substitutions: a density elimination method that, similarly to normalization for natural deduction systems, removes applications of $(D)$ by making suitable substitutions in the proof for the introduced variables. Proceeding "by substitutions" instead of shifting applications of $(D)$ upwards in the proof (as e.g. in $[2,10]$ ) avoids the need for more complicated combinatorial ("Gentzen's mix"-style) rules as induction hypotheses.

First some notation. Let HL be any (hyper)sequent calculus. $d, S_{1}, \ldots, S_{n} \vdash_{\mathbf{H L}} S$ stands for a derivation $d$ in $\mathbf{H L}$ of the (hyper)sequent $S$ from the assumptions $S_{1}, \ldots, S_{n}$. Let $H$ be a hypersequent. $H\left[{ }^{\Sigma} / p^{l}, \Gamma \Rightarrow \Delta / p^{r}\right]$ is the hypersequent obtained by replacing in $H$ all the left occurrences of $p$ with $\Sigma$ and all the components $\Pi \Rightarrow p$ with $\Pi, \Gamma \Rightarrow \Delta . d(s)$ and $H(s)$ denote the results of substituting the term $s$ for all free occurrences of $x$ in the derivation $d(x)$ and in the (hyper)sequent $H(x)$, respectively. The length $|d|$ of a derivation $d$ in $\mathbf{H L}$ is (the maximal number of inference rules occurring on any branch of $d)+1$.

We require the following crucial lemma (proved by easy inductions on the lengths of derivations) asserting the "substitutivity" of calculi with substitutive rules:

Lemma 1. Let $\mathbf{L}_{\mathbf{L}}$ be a (first-order) simple sequent calculus with substitutive rules:

(1) If $d_{1}(x) \vdash_{\mathbf{H L}_{\mathbf{L}}^{\mathbf{C}}} H(x)$, then $d_{1}(y) \vdash_{\mathbf{H L}_{\mathbf{L}}^{\mathbf{C}}} H(y)$ where $y$ does not occur in $d_{1}(x)$.

(2) If $\vdash_{\mathbf{H L}_{\mathbf{L}}^{\mathbf{C}}} H$, then $\vdash_{\mathbf{H L}_{\mathbf{L}}^{\mathbf{C}}} H\left[{ }^{A} / p^{l},{ }^{A} / p^{r}\right]$ for any formula $A$ and propositional variable $p$.

Our density elimination method proceeds by removing applications of $(D)$ which are topmost in the proof. Let, e.g. $d$ be:

$$
\frac{\vdots d^{\prime}}{G|\Gamma \Rightarrow p| \Sigma, p \Rightarrow \Delta}
$$


a subderivation ending in such an application of $(D)$. The idea is to replace the occurrences of $p$ in $d$ in an "asymmetric" way, according to whether $p$ occurs in the antecedent or consequent of a sequent (component of the hypersequent). Roughly speaking, in $d$ each sequent $\Pi, \underline{p} \Rightarrow \Pi^{\prime}$ is replaced by $\Pi, \underline{\Gamma} \Rightarrow \Pi^{\prime}$ and each $\Pi \Rightarrow \underline{p}$ by $\Pi, \underline{\Sigma} \Rightarrow \underline{\Delta}$. (Note that condition $(*)$ in Definition 1 prevents $p$ 's from jumping from one side of a sequent in a rule's premise, to the other in the rule's conclusion.) The resulting tree is then transformed into a density-free derivation by replacing:

(a) the application of $(D)$ above by $(E C)$.

(b) each application of a substitutive rule by suitable inferences.

(c) each subproof ending in an application of $(C O M)$ and containing one occurrence of the axiom $p \Rightarrow p$, as e.g. in

$$
\frac{G\left|\Gamma^{\prime}, \Pi \Rightarrow d_{1} \quad G\right| \Gamma, \Sigma \Rightarrow \Delta}{G\left|\Gamma, \Pi \Rightarrow \Pi^{\prime}\right| \Gamma^{\prime}, \Sigma \Rightarrow \Delta}
$$

by a suitable derivation of the form

$$
\begin{gathered}
\vdots d_{1} \\
\frac{G \mid \Gamma^{\prime}, \Pi \Rightarrow \Pi^{\prime}}{\vdots} \\
G\left|\Gamma, \Pi \Rightarrow \Pi^{\prime}\right| \Gamma^{\prime}, \Sigma \Rightarrow \Delta
\end{gathered}
$$

Theorem 2 (Density Elimination). Let $\mathbf{L}_{\mathbf{L}}$ be a (first-order) $w$-simple sequent calculus whose rules are reductive and substitutive. If $\mathbf{L}_{\mathbf{L}}$ includes the rules $(\odot, l)$ and $(\odot, r)$ in Fig. 1 then $\mathbf{H L}_{\mathbf{L}}^{\mathbf{C}}$ plus $(D)$ admits density elimination.

Proof. W.l.o.g. consider the above (sub)derivation $d$ in $\mathbf{H L}_{\mathbf{L}}^{\mathbf{C}}$ plus $(D)$ ending in a topmost application of $(D)$. By Theorem 1 we can assume that $d$ is cut-free. We first show that for each hypersequent $H$ in $d^{\prime}$ in which no component has the form $\Pi, p^{j} \Rightarrow$ $p$, with $j \geq 1$, one can find $d_{H}^{\prime}$ such that

$$
d_{H}^{\prime} \vdash_{\mathbf{H L}_{\mathbf{L}}^{\mathbf{C}}} G \mid H\left[{ }^{\Gamma} / p^{l},{ }^{\Sigma \Rightarrow \Delta} / p^{r}\right]
$$

The proof proceeds by induction on the length of the cut-free derivation $d_{H}$ of $H$ in $\mathbf{H L}_{\mathbf{L}}^{\mathbf{C}}$. We distinguish cases according to the last rule $(r)$ applied in $d_{H}$.

- If $\left|d_{H}\right|=0$, i.e. $H$ is $G^{\prime} \mid B \Rightarrow B$, then the claim holds by applying $(E W)$.

- If $(r)$ is $(E C)$ or $(E W)$, then the claim follows by the i.h. and applying $(r)$.

- Let $(r)$ be a rule other than $(E C),(E W)$, or $(C O M)$, w.l.o.g. of the form:

$$
\frac{G^{\prime}\left|S_{1} \ldots G^{\prime}\right| S_{m}}{G^{\prime} \mid S}
$$

Since $G^{\prime} \mid S$ does not contain any component of the form $\Pi, p^{j} \Rightarrow p$, with $j \geq 1$, by condition $(*)$ in Def. 1 and the absence of cuts, no $G^{\prime} \mid S_{i}$ (with $i \in\{1, \ldots, m\}$ ) contains any $\Pi, p^{j} \Rightarrow p$, with $j \geq 1$. Hence by the i.h.:

$$
\vdash_{\mathbf{H L}_{\mathbf{L}}^{\mathbf{C}}} G\left|\left(G^{\prime} \mid S_{1}\right)\left[{ }^{\Gamma} / p^{l},{ }^{\Sigma \Rightarrow \Delta} / p^{r}\right] \quad \ldots \quad \vdash_{\mathbf{H L}_{\mathbf{L}}^{\mathbf{C}}} G\right|\left(G^{\prime} \mid S_{m}\right)\left[\Gamma / p^{l},,^{\Sigma \Rightarrow \Delta} / p^{r}\right]
$$


Since the rules of $\mathbf{L}_{\mathbf{L}}$ are substitutive, using Lemma 1.(1) to take care of renaming variables, there exists a derivation for:

$$
S_{1}\left[{ }^{\Gamma} / p^{l},{ }^{\Sigma} \Rightarrow \Delta / p^{r}\right], \ldots, S_{m}\left[\Gamma / p^{l},{ }^{\Sigma} \Rightarrow \Delta / p^{r}\right] \vdash_{\mathbf{L}_{\mathbf{L}}} S\left[{ }^{\Gamma} / p^{l},{ }^{\Sigma \Rightarrow \Delta} / p^{r}\right]
$$

that uses only the structural rules of $\mathbf{L}_{\mathbf{L}}$ and (possibly) $(r)$. The claim then follows by $(E W)$, lifting the above derivation from $\mathbf{L}_{\mathbf{L}}$ to $\mathbf{H L}_{\mathbf{L}}$.

- If $(r)$ is $(C O M)$, two cases can occur: (a) none of its premises contains any $\Pi, p^{j} \Rightarrow p$, with $j \geq 1$ or (b) one of the premises does. For (a), the claim holds by applying the i.h. followed by an application of $(C O M)$. As an example, consider the application of $(C O M)$ :

$$
\begin{array}{cc}
\vdots & \vdots \\
G_{1} \mid \Gamma^{\prime}, \Pi \Rightarrow p & G^{\prime} \mid \Sigma^{\prime}, p^{k}, \Pi^{\prime} \Rightarrow B \\
\hline G^{\prime}\left|\Gamma^{\prime}, \Sigma^{\prime}, p^{k} \Rightarrow B\right| \Pi, \Pi^{\prime} \Rightarrow p & (\text { (Со) })
\end{array}
$$

Let $G^{*}=G^{\prime}\left[\Gamma / p^{l},{ }^{\Sigma} \Rightarrow \Delta / p^{r}\right]$. By the i.h.:

$$
\vdash_{\mathbf{H L}_{\mathbf{L}}^{\mathbf{C}}} G\left|G^{*}\right| \Gamma^{\prime}, \Pi, \Sigma \Rightarrow \Delta \quad \text { and } \vdash_{\mathbf{H L}_{\mathbf{L}}^{\mathbf{C}}} G\left|G^{*}\right| \Sigma^{\prime}, \Gamma^{k}, \Pi^{\prime} \Rightarrow B
$$

Hence by $(C O M), \vdash_{\mathbf{H L}_{\mathrm{L}}^{\mathrm{C}}} G\left|G^{*}\right| \Gamma^{\prime}, \Sigma^{\prime}, \Gamma^{k} \Rightarrow B \mid \Pi, \Pi^{\prime}, \Sigma \Rightarrow \Delta$.

For (b), we have an application of $(C O M)$ :

$$
\frac{G^{\prime}\left|\Gamma^{\prime}, \Pi, p^{l} \Rightarrow p \quad G^{\prime}\right| \Sigma^{\prime}, p^{(k-l)}, \Pi^{\prime} \Rightarrow B}{G^{\prime}\left|\Gamma^{\prime}, \Sigma^{\prime}, p^{k} \Rightarrow B\right| \Pi, \Pi^{\prime} \Rightarrow p}
$$

Letting again $G^{*}=G^{\prime}\left[\Gamma / p^{l}, \Sigma \Rightarrow \Delta / p^{r}\right]$, by the i.h.:

$$
d_{1} \vdash_{\mathbf{H L}_{\mathbf{L}}^{\mathbf{C}}} G\left|G^{*}\right| \Sigma^{\prime}, \Gamma^{(k-l)}, \Pi^{\prime} \Rightarrow B .
$$

Our aim is to show $\vdash_{\mathbf{H L}_{\mathrm{L}}^{\mathrm{C}}} G\left|G^{*}\right| \Gamma^{\prime}, \Sigma^{\prime}, \Gamma^{k} \Rightarrow B \mid \Pi, \Pi^{\prime}, \Sigma \Rightarrow \Delta$. If $\Pi^{\prime}=\emptyset$, then the result follows by $(w, l)$ so assume that $\Pi^{\prime}=A_{1}, \ldots, A_{m}$. Let $d_{1}^{\prime}$ be the derivation obtained by $m$ applications of $(\odot, l)$ to the end-hypersequent of $d_{1}$, i.e.

$$
\begin{aligned}
& \vdots d_{1} \\
& \frac{G\left|G^{*}\right| \Sigma^{\prime}, \Gamma^{(k-l)}, \Pi^{\prime} \Rightarrow B}{G\left|G^{*}\right| \Sigma^{\prime}, \Gamma^{(k-l)}, A_{1} \odot \ldots \odot A_{m} \Rightarrow B}{ }^{(\odot, 1) \times \mathrm{m}}
\end{aligned}
$$

Denote $\left(A_{1} \odot \ldots \odot A_{m}\right)$ by $\odot \Pi^{\prime}$. Consider the proof $d^{\prime}$ above ending in the premise $G|\Gamma \Rightarrow p| \Sigma, p \Rightarrow \Delta$ of the $(D)$ rule. By Lemma 1.(2), $d_{2} \vdash_{\mathbf{H L}_{\mathbf{L}}^{\mathbf{C}}}(G \mid \Gamma \Rightarrow$ $p \mid \Sigma, p \Rightarrow \Delta)\left[\odot \Pi^{\prime} / p^{l}, \odot \Pi^{\prime} / p^{r}\right]$ for some derivation $d_{2}$, that is:

$$
d_{2} \vdash_{\mathbf{H L}_{\mathbf{L}}^{\mathbf{C}}} G\left|\Gamma \Rightarrow \odot \Pi^{\prime}\right| \Sigma, \odot \Pi^{\prime} \Rightarrow \Delta .
$$

The desired derivation is then obtained by applying (CUT) to the easily derived sequent $\Pi^{\prime} \Rightarrow \odot \Pi^{\prime}$ and the end sequent of the following derivation:

$$
\begin{aligned}
& \vdots d_{2}
\end{aligned}
$$

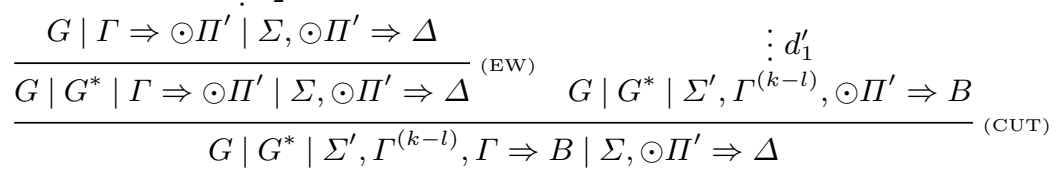


with subsequent applications of $(w, l)$.

Finally, let $H$ be the premise $G|\Gamma \Rightarrow p| \Sigma, p \Rightarrow \Delta$ of the $(D)$ rule in $d$. We have shown that $\vdash_{\mathbf{H L}_{\mathbf{L}}^{\mathrm{C}}} G|G| \Gamma, \Sigma \Rightarrow \Delta \mid \Gamma, \Sigma \Rightarrow \Delta$. (Note that $G\left[{ }^{\Gamma} / p^{l},{ }^{\Sigma \Rightarrow \Delta} / p^{r}\right]=G$ ). The desired proof of $G \mid \Gamma, \Sigma \Rightarrow \Delta$ follows by multiple applications of $(E C)$.

Theorem 3. Let $\mathbf{L}_{\mathbf{L}}$ be a (first-order) $w$-simple sequent calculus whose rules are reductive and substitutive. $\mathbf{H L}_{\mathbf{L}}^{\mathbf{C}}$ plus $(D)$ admits cut elimination and density elimination.

Proof. Let $\star$ be a new 2-ary connective. Let $\mathbf{L}_{\mathbf{L}}^{\prime}$ be $\mathbf{L}_{\mathbf{L}}$ extended with the following (reductive and substitutive) rules:

$$
\frac{\Theta, X, Y \Rightarrow \Xi}{\Theta, X \star Y \Rightarrow \Xi}(\star, l) \quad \frac{\Theta \Rightarrow Y \quad \Theta^{\prime} \Rightarrow X}{\Theta, \Theta^{\prime} \Rightarrow X \star Y}(\star, r)
$$

$\mathbf{H L}_{\mathbf{L}}^{\prime \mathbf{C}}$ admits cut elimination by Theorem 1 . Also, as $\star$ has the rules of $\odot, \mathbf{H L}_{\mathbf{L}}^{\prime \mathbf{C}}$ plus $(D)$ admits density elimination by Theorem 2 . Then since $\mathbf{H L}_{\mathbf{L}}^{\prime \mathbf{C}}$ has the subformula property, $\mathbf{H L}_{\mathbf{L}}^{\mathbf{C}}$ plus $(D)$ admits cut elimination and density elimination.

\section{Axiomatizations}

We use density elimination to establish so-called "rational completeness" for a wide class of first-order substructural logics described below. Our vocabulary is assumed to include the binary connectives $\wedge, \vee, \odot, \rightarrow$; the constants $f, t, \top, \perp$; and the defined connective $A \leftrightarrow B==_{\text {def }}(A \rightarrow B) \wedge(B \rightarrow A)$. A logic $\mathbf{L}$ is treated as a Hilbert system, where $T \vdash_{\mathbf{L}} A$ if there exists a derivation (in the usual sense) of a formula $A$ from a set of formulae $T$ in $\mathbf{L}$. We begin by recalling the (propositional) Uninorm logic $\mathbf{U L}$ of [10] (an axiomatization for $\mathbf{F L}_{e}$ plus (L5)), given by the axiom schema:
(L1) $X \rightarrow X$
(L2) $(X \rightarrow Y) \rightarrow((Y \rightarrow Z) \rightarrow(X \rightarrow Z))$
(L8) $X \rightarrow(X \vee Y)$
(L3) $(X \rightarrow(Y \rightarrow Z)) \rightarrow(Y \rightarrow(X \rightarrow Z))$
(L9) $Y \rightarrow(X \vee Y)$
(L4) $((X \odot Y) \rightarrow Z) \leftrightarrow(X \rightarrow(Y \rightarrow Z))$
(L10) $(X \wedge Y) \rightarrow Y$
(L5) $((X \rightarrow Y) \wedge t) \vee((Y \rightarrow X) \wedge t)$
(L11) $(X \wedge Y) \rightarrow X$
(L6) $((X \rightarrow Z) \wedge(Y \rightarrow Z)) \rightarrow((X \vee Y) \rightarrow Z)$
(L12) $X \leftrightarrow(t \rightarrow X)$
(L7) $((X \rightarrow Y) \wedge(X \rightarrow Z)) \rightarrow(X \rightarrow(Y \wedge Z))$
(L13) $\perp \rightarrow X$
(L14) $X \rightarrow \top$

together with the inference rules:

$$
\frac{X \quad X \rightarrow Y}{Y}(m p) \quad \frac{X \quad Y}{X \wedge Y}(a d j)
$$

Definition 7. We call any logic $\mathbf{L}$ resulting from $\mathbf{U L}$ by adding "extra" propositional axioms (possibly with "extra" connectives), and satisfying for all formulae $A, B, C$ :

$$
A \leftrightarrow B \vdash_{\mathbf{L}} C(A) \leftrightarrow C(B)
$$

$a$ core UL-expansion. ${ }^{4}$

\footnotetext{
${ }^{4}$ A related notion of a "core fuzzy logic", restricted to logics with weakening, is used in [5].
} 
Definition 8. For any core $\mathbf{U L}$-expansion $\mathbf{L}, \forall \mathbf{L}$ consists of $\mathbf{L}$ plus:

$$
\begin{gathered}
(\forall 1) \forall x Y \rightarrow Y(x / t) \quad(t \text { substitutable for } x \text { in } Y) \\
(\forall 2) \forall x(Y \rightarrow Z) \rightarrow(Y \rightarrow \forall x Z) \quad \text { (x not free in } Y) \\
(\forall 3) \forall x(Y \vee Z) \rightarrow(Y \vee \forall x Z) \quad(x \text { not free in } Y) \\
(\exists 1) Y(x / t) \rightarrow \exists x Y \quad(t \text { substitutable for } x \text { in } Y) \\
(\exists 2) \forall x(Y \rightarrow Z) \rightarrow(\exists x Y \rightarrow Z) \quad(x \text { not free in } Z) \\
\frac{Y}{\forall x Y}(\text { gen })
\end{gathered}
$$

Semantics for these logics are based on pointed bounded commutative residuated lattices: algebras $\langle L, \wedge, \vee, \odot, \rightarrow, t, f, \perp, \top\rangle$ with binary operations $\wedge, \vee, \odot, \rightarrow$, and constants $t, f, \perp$, $\top$ such that $\langle L, \wedge, \vee, \perp, \top\rangle$ is a bounded lattice; $\langle L, \odot, t\rangle$ is a commutative monoid; and $z \leq x \rightarrow y$ iff $x \odot z \leq y$ for all $x, y, z \in L$.

A $U L$-algebra is a pointed bounded commutative residuated lattice satisfying:

$$
t \leq((x \rightarrow y) \wedge t) \vee((y \rightarrow x) \wedge t)
$$

If $\mathcal{A}$ is an algebra such that each connective of a language $\mathcal{L}$ occurs as an operation of $\mathcal{A}$, then $(\mathcal{L}$-)valuations for $\mathcal{A}$ with (non-empty) domain $\mathcal{D}$ are defined as partial functions $v$ from sentences of $\mathcal{L}$ with parameters in $\mathcal{D}$ into $\mathcal{A}$, total on atomic sentences, where:

1. If $v\left(A_{i}\right)$ is defined for $i=1 \ldots m$, then $v\left(\star\left(A_{1}, \ldots, A_{m}\right)\right)=\star\left(v\left(A_{1}\right), \ldots, v\left(A_{m}\right)\right)$ for each $m$-ary connective $\star$ of $\mathcal{L}$.

2. If for all $c \in \mathcal{D}, v(A(x / c))$ is defined and $\inf (\{v(A(x / c)): c \in \mathcal{D}\})$ exists, then $v(\forall x A)=\inf (\{v(A(x / c)): c \in \mathcal{D}\})$.

3. If for all $c \in \mathcal{D}, v(A(x / c))$ is defined and $\sup (\{v(A(x / c)): c \in \mathcal{D}\})$ exists, then $v(\exists x A)=\sup (\{v(A(x / c)): c \in \mathcal{D}\})$.

A valuation $v$ for $\mathcal{A}$ with domain $\mathcal{D}$ is safe if $v(A)$ is defined for every sentence with parameters in $\mathcal{D}$. A formula $A$ is valid in $\mathcal{A}$ (assuming that $\mathcal{A}$ contains the constant $t$ ) iff for every non-empty set $\mathcal{D}$ and for every safe valuation $v$ with domain $\mathcal{D}, v(A) \geq t$.

Definition 9. For a core $\mathbf{U L}$-expansion $\mathbf{L}$ with extra connectives $I$, an $\mathbf{L}$-algebra is an algebra $\mathcal{A}=\left\langle L, \wedge, \vee, \odot, \rightarrow, t, f, \perp, \top,(c)_{c \in I}\right\rangle$ such that $\langle L, \wedge, \vee, \odot, \rightarrow, t, f, \perp, \top\rangle$ is a UL-algebra and each additional axiom of $\mathbf{L}$ is valid in $\mathcal{A}$. An L-algebra is called an L-chain if it is linearly ordered, and a dense L-chain if it is linearly and densely ordered.

Recall that a first-order theory $T$ is a set of sentences:

1. $T$ is linear if for each pair $A, B$ of sentences, either $A \rightarrow B \in T$ or $B \rightarrow A \in T$.

2. $T$ is $\forall \mathbf{L}$-dense if for each pair $A, B$ of sentences, whenever $T \nvdash_{\forall \mathbf{L}} A \rightarrow B$, then for some sentence $C, T \nvdash_{\forall \mathbf{L}} A \rightarrow C$ and $T \nvdash_{\forall \mathbf{L}} C \rightarrow B$.

3. $T$ is $\forall \mathbf{L}$-Henkin if for each sentence of the form $\forall x A(x)$ where $T \nvdash_{\forall \mathbf{L}} \forall x A(x)$, there is a constant $c$ in the language of $T$ such that $T \nvdash_{\forall \mathbf{L}} A(c)$.

The Lindenbaum algebra of a theory $T$ is defined as follows, justified by the fact that the connective $\leftrightarrow$ has the property of a "congruence" for any core UL-expansion: 
Definition 10. Let $\mathbf{L}$ be a core $\mathbf{U L}$-expansion $\mathbf{L}$ and $T$ a theory. The Lindenbaum algebra $\mathcal{A}_{T}^{\forall \mathrm{L}}$ of a theory $T$ has universe $L_{T}=\left\{[A]_{T}:\right.$ A a sentence $\}$ where $[A]_{T}=$ $\left\{B: T \vdash_{\forall \mathbf{L}} A \leftrightarrow B\right\}$, and operations $\star\left(\left[A_{1}\right]_{T}, \ldots,\left[A_{n}\right]_{T}\right)=\left[\star\left(A_{1}, \ldots, A_{n}\right)\right]$ for each n-ary connective $\star$ of $\mathbf{L}$.

The proof of the following lemma then proceeds exactly as for Lemma 5.2.6 in [8]:

Lemma 2. Let $\mathbf{L}$ be a core $\mathbf{U L}$-expansion $\mathbf{L}$ and $T$ a $\forall \mathbf{L}$-Henkin theory. Then for any formula $A(x)$ with one free variable $x:[\forall x A]_{T}=\inf _{c}[A(c)]_{T}$ and $[\exists x A]_{T}=$ $\sup _{c}[A(c)]_{T}$ where c runs over all constants of $T$.

\section{Adding Density}

Here we show that adding the density rule to any first-order core $\mathbf{U L}$-expansion $\mathbf{L}$ gives a logic that is complete with respect to dense $L$-chains. To add the density rule to our axiomatizations, we explicitly define derivations from a set of formulae $T$ to take account of the fact that applications of (density) are sensitive to $T$ and might therefore require new propositional variables.

Definition 11. For $\mathbf{L}$ a core $\mathbf{U L}$-expansion, let $\forall \mathbf{L}^{\mathbf{D}}$ be $\forall \mathbf{L}$ extended with the rule (density) (see Section 1). $A \forall \mathbf{L}^{\mathbf{D}}$-derivation of a formula $C$ from a set of formulae $T$, written $T \vdash_{\forall \mathbf{L}^{\mathbf{D}}} C$, consists of a sequence of formulae in the vocabulary of $\forall \mathbf{L}^{\mathbf{D}}$ extended with countably many new constants and propositional variables, ending with $C$ and such that each member $A$ of the sequence satisfies one of the following:

(1) $A \in T$ or $A$ is an axiom of $\forall \mathbf{L}$ for the extended language.

(2) A is obtained from previous members of the sequence by (mp), (adj), or (gen) i.e. either $B$ and $B \rightarrow A, B$ and $C$ (where $A$ is $B \wedge C$ ), or $B(a)$ (where $A$ is $\forall x B(x)$ and the eigenvariable condition for $T$ is satisfied) occur earlier in the sequence.

(3) $A$ is obtained from a previous member of the sequence by (density), i.e. $A$ is $(B \rightarrow C) \vee D$ and $(B \rightarrow p) \vee(p \rightarrow C) \vee D$ occurs earlier in the sequence, where $p$ is a new propositional variable not occurring in $T, B, C$, or $D$.

Soundness for $\forall \mathbf{L}^{\mathbf{D}}$ with respect to dense L-chains is established as follows.

Lemma 3. Let $\mathbf{L}$ be a core $\mathbf{U L}$-expansion. If $T \vdash_{\forall \mathbf{L}^{\mathrm{D}}} A$, then for every dense L-chain and safe valuation $v$ with non-empty domain $\mathcal{D}$, if $v(B) \geq t$ for all $B \in T$, then $v(A) \geq t$. In particular, if $\vdash_{\forall \mathbf{L}^{\mathrm{D}}} A$, then $A$ is valid in all dense L-chains.

Proof. We proceed by induction on the height of a $\forall \mathbf{L}^{\mathbf{D}}$-derivation for $T \vdash_{\forall \mathbf{L}^{\mathbf{D}}} A$, checking the validity of axioms and soundness of rules in the usual manner, the only novel case being (density). Suppose contrapositively that there is a dense L-chain and non-empty domain $\mathcal{D}$ with a safe valuation $v$ such that $v(D) \geq t$ for all $D \in T$, and $v((A \rightarrow B) \vee C)<t$. It follows that $v(A \rightarrow B)<t$ and $v(C)<t$, and hence also that $v(A)>v(B)$. Since the algebra is dense, there exists an element $w$ such that $v(A)>w>v(B)$. Recall that the propositional variable $p$ in (density) does not occur in $T, A, B$, or $C$. Hence we can extend the valuation $v$ with $v(p)=w$. It follows that $v((A \rightarrow p) \vee(p \rightarrow B) \vee C)<t$. 
We require the following properties (established as in the propositional case in [10]):

Lemma 4. Let $\mathbf{L}$ be a core $\mathbf{U L}$-expansion:

(a) If $T, A \vdash_{\forall \mathbf{L}^{\mathrm{D}}} C$ and $T, B \vdash_{\forall \mathbf{L}^{\mathrm{D}}} C$, then $T, A \vee B \vdash_{\forall \mathbf{L}^{\mathrm{D}}} C$.

(b) If $T, A \vdash_{\forall \mathbf{L}^{\mathrm{D}}} C$ and $T \vdash_{\forall \mathbf{L}^{\mathrm{D}}} A \vee C$, then $T \vdash_{\forall \mathbf{L}^{\mathrm{D}}} C$.

Definition 12. A confusion of a set of formulae $T$ is defined inductively as follows:

1. $t, T$, and any element of $T$ are confusions of $T$.

2. If $C_{1}$ and $C_{2}$ are confusions of $T$, then so are $C_{1} \odot C_{2}$ and $C_{1} \wedge C_{2}$.

Lemma 5. Let $\mathbf{L}$ be a core $\mathbf{U L}$-expansion:

(a) If $T \vdash_{\forall \mathbf{L}^{\mathrm{D}}} A$, then $T_{0} \vdash_{\forall \mathbf{L}^{\mathrm{D}}}$ A for some finite subset $T_{0}$ of $T$.

(b) If $T$ is finite, then $T \vdash_{\forall \mathbf{L}^{\mathrm{D}}} A$ iff $\vdash_{\forall \mathbf{L}^{\mathrm{D}}} C \rightarrow A$ for some confusion $C$ of $T$.

(c) If $A$ is a confusion of $T$, then $T \vdash_{\forall \mathbf{L}} A$.

We are now in a position to establish our key lemmas.

Lemma 6. Let $\mathbf{L}$ be a core $\mathbf{U L}$-expansion and $T$ a countable theory. If $T \forall_{\forall \mathbf{L}^{\mathrm{D}}} A$, then there exists a countable linear $\forall \mathbf{L}$-dense $\forall \mathbf{L}$-Henkin theory $\hat{T}$ such that $T \subseteq \hat{T}$ and $\hat{T} \nvdash_{\forall \mathbf{L}^{\mathrm{D}}} A$.

Proof. We construct $\hat{T}$ in countably many steps. First we extend the vocabulary with countably many new propositional variables and constants not occurring in $T$ or $A$. In the construction of $\hat{T}$ we have to: (1) deal with linearity and $\forall \mathbf{L}$-density for each pair of sentences (in the extended vocabulary), and (2) deal with the $\forall \mathbf{L}$-Henkin property for each sentence of the form $\forall x A$ (in the extended vocabulary). Since these are countably many tasks we can interleave them.

We begin by defining $T_{0}=T$ and $C_{0}=A$, noting that $T_{0} \nvdash_{\forall \mathbf{L}^{\mathrm{D}}} C_{0}$. Now for the induction step, assume that $T_{n}$ and $C_{n}$ have been constructed such that $T_{n} \nvdash_{\forall \mathbf{L} \mathrm{D}} C_{n}$. We construct $T_{n+1}$ and $C_{n+1}$ such that $T_{n} \subseteq T_{n+1} ; T_{n+1} \nvdash_{\forall \mathbf{L}^{\mathrm{D}}} C_{n+1} ; T_{n+1} \vdash_{\forall \mathbf{L}^{\mathrm{D}}}$ $C_{n} \rightarrow C_{n+1}$; and $T_{n+1}$ fulfills the $n$-th task. We have two cases:

(1) The $n$-th task is dealing with linearity and $\forall \mathbf{L}$-density for the sentences $A, B$.

- If $T_{n} \cup\{A \rightarrow B, B \rightarrow A\} \nvdash_{\forall \mathbf{L}^{\mathrm{D}}} C_{n}$, then it is sufficient to define:

$$
T_{n+1}=T_{n} \cup\{A \rightarrow B, B \rightarrow A\} \text { and } C_{n+1}=C_{n}
$$

- Suppose that the previous case does not apply. Let $q$ be a propositional variable not occurring in $T_{n}, A, B$, or $C_{n}$. We claim that one of the following conditions holds:

(a) $T_{n} \cup\{A \rightarrow B\} \nvdash_{\forall \mathbf{L}^{\mathrm{D}}} C_{n} \vee(B \rightarrow q) \vee(q \rightarrow A)$

(b) $T_{n} \cup\{B \rightarrow A\} \forall \forall \forall \mathbf{L}^{\mathrm{D}} C_{n} \vee(A \rightarrow q) \vee(q \rightarrow B)$.

Suppose that (a) does not hold. Then by the density rule:

$$
T_{n} \cup\{A \rightarrow B\} \vdash_{\forall \mathbf{L}^{\mathrm{D}}} C_{n} \vee(B \rightarrow A)
$$

and since $T_{n} \cup\{A \rightarrow B, B \rightarrow A\} \vdash_{\forall \mathbf{L}^{\mathrm{D}}} C_{n}$, by Lemma 4 (b):

$$
T_{n} \cup\{A \rightarrow B\} \vdash_{\forall \mathbf{L}^{\mathrm{D}}} C_{n}
$$


If (b) also does not hold, $T_{n} \cup\{B \rightarrow A\} \vdash_{\forall \mathbf{L}^{\mathrm{D}}} C_{n}$, so by Lemma 4 (a):

$$
T_{n} \cup\{(A \rightarrow B) \vee(B \rightarrow A)\} \vdash_{\forall \mathbf{L}^{\mathrm{D}}} C_{n}
$$

But since $\vdash_{\mathbf{U L}}(A \rightarrow B) \vee(B \rightarrow A)$ (see e.g. [10]), we get $T_{n} \vdash_{\mathbf{L}^{\mathrm{D}}} C_{n}$ which contradicts the induction hypothesis. Hence, if (a) holds, let:

$$
T_{n+1}=T_{n} \cup\{A \rightarrow B\} \text { and } C_{n+1}=C_{n} \vee(B \rightarrow q) \vee(q \rightarrow A)
$$

and if (b) holds, let:

$$
T_{n+1}=T_{n} \cup\{B \rightarrow A\} \text { and } C_{n+1}=C_{n} \vee(A \rightarrow q) \vee(q \rightarrow B)
$$

Clearly $T_{n+1}$ is linear in both cases, $T_{n+1} \nvdash_{\forall \mathbf{L}^{\mathrm{D}}} C_{n+1}$, and $T_{n+1} \vdash_{\forall \mathbf{L}^{\mathrm{D}}} C_{n} \rightarrow$ $C_{n+1}$. Moreover, if $T_{n+1} \nvdash_{\forall \mathbf{L}} A \rightarrow B$, then $T_{n+1} \nvdash_{\forall \mathbf{L}^{\mathrm{D}}} C_{n} \vee(A \rightarrow q) \vee(q \rightarrow B)$, so $T_{n+1} \nvdash_{\forall \mathbf{L}} A \rightarrow q$ and $T_{n+1} \nvdash_{\forall \mathbf{L}} q \rightarrow B$. Similarly, if $T_{n+1} \nvdash_{\forall \mathbf{L}} B \rightarrow A$, then $T_{n+1} \nvdash_{\forall \mathbf{L}} B \rightarrow q$ and $T_{n+1} \nvdash_{\forall \mathbf{L}} q \rightarrow A$, so $\forall \mathbf{L}$-density holds.

(2) Suppose that the $n$-th task is dealing with the $\forall \mathbf{L}$-Henkin property for $\forall x A(x)$. Let $c$ be a new constant not occurring in $T_{n}, C_{n}$, or $A(x)$.

- If $T_{n} \nvdash_{\forall \mathbf{L}} C_{n} \vee A(c)$, then $T_{n} \nvdash_{\forall \mathbf{L}} \forall x A(x)$, and let $T_{n+1}=T_{n}$, and $C_{n+1}=$ $C_{n} \vee A(c)$.

- If $T_{n} \vdash_{\forall \mathbf{L}} C_{n} \vee A(c)$, then $T_{n} \vdash_{\forall \mathbf{L}} C_{n} \vee A(x)$ (replacing $c$ in the proof by a variable $x$ not occurring in $\left.C_{n}\right)$. Hence $T_{n} \vdash_{\forall \mathbf{L}} \forall x\left(C_{n} \vee A(x)\right)$ by $(g e n)$, and by $(\forall 3), T_{n} \vdash_{\forall \mathbf{L}} C_{n} \vee \forall x A(x)$. So $T_{n} \cup\left\{\forall x A(x) \rightarrow C_{n}\right\} \vdash_{\forall \mathbf{L}} C_{n}$. It follows that $T_{n} \cup\left\{C_{n} \rightarrow \forall x A(x)\right\} \quad \forall_{\forall \mathbf{L}} C_{n}$ (since otherwise $T_{n} \vdash_{\forall \mathbf{L}^{\mathrm{D}}} C_{n}$ a contradiction) and $T_{n} \cup\left\{C_{n} \rightarrow \forall x A(x)\right\} \vdash_{\forall \mathbf{L}} \forall x A(x)$ (using $T_{n} \vdash_{\forall \mathbf{L}} C_{n} \vee A(c)$ ). So let $T_{n+1}=T_{n} \cup\left\{C_{n} \rightarrow \forall x A(x)\right\}$ and $C_{n+1}=C_{n}$.

Now take $\hat{T}=\bigcup_{n \in \mathbb{N}} T_{n} . \hat{T}$ is linear and $\forall \mathbf{L}$-dense by construction. Also $\hat{T} \nvdash_{\forall \mathbf{L}^{\mathrm{D}}} A$ since otherwise $T_{n} \vdash_{\forall \mathbf{L}^{\mathrm{D}}} A$ for some $n$, and since $T_{n} \vdash_{\forall \mathbf{L}^{\mathrm{D}}} C_{i} \rightarrow C_{i+1}$ for $i=$ $1 \ldots n, T_{n} \vdash_{\forall \mathbf{L}^{\mathrm{D}}} C_{n}$, a contradiction. Finally to see that $\hat{T}$ is $\forall \mathbf{L}$-Henkin, suppose that $\hat{T} \nvdash_{\forall \mathbf{L}} \forall x A(x)$ where $\forall x A(x)$ is a sentence dealt with in step $n$. It follows that $T_{n+1} \not \forall \mathbf{L} \forall x A(x)$. But then for step $n$ in the above construction, we must have the first possibility $T_{n} \nvdash_{\forall \mathbf{L}} C_{n} \vee A(c)$ where $C_{n+1}=C_{n} \vee A(c)$. Hence also $\hat{T} \nvdash_{\forall \mathbf{L}} C_{n} \vee A(c)$. So $\hat{T} \nvdash_{\forall \mathbf{L}} A(c)$.

Lemma 7. Let $\mathbf{L}$ be a core $\mathbf{U L}$-expansion and $T$ a countable linear $\forall \mathbf{L}$-dense $\forall \mathbf{L}$ Henkin theory. If $T \forall_{\forall \mathbf{L}^{\mathrm{D}}} A$, then there exists a countable dense L-chain and safe valuation $v$ with non-empty domain $\mathcal{D}$, such that $v(B) \geq t$ for all $B \in T$, and $v(A)<t$.

Proof. $\mathcal{A}_{T}^{\forall \mathrm{L}}$ is an L-algebra. Moreover, $\mathcal{A}_{T}^{\forall \mathrm{L}}$ is linearly and densely ordered, since for all sentences $A, B$ : (1) either $A \rightarrow B \in T$ or $B \rightarrow A \in T$, and (2) if $T \nvdash_{\forall \mathbf{L}} A \rightarrow B$, then $T \nvdash_{\forall \mathbf{L}} A \rightarrow C$ and $T \nvdash_{\forall \mathbf{L}} C \rightarrow B$ for some sentence $C$. Let $\mathcal{D}$ be the set of all constants of the vocabulary of $T$ (adding one if necessary so that $\mathcal{D}$ is non-empty) and $A$. Define a valuation $v$ with domain $\mathcal{D}$ such that $v\left(p\left(c_{1}, \ldots, c_{m}\right)\right)=\left[p\left(c_{1}, \ldots, c_{m}\right)\right]_{T}$ for each $m$-ary predicate $p$. We claim that $v(B)=[B]_{T}$ for all formulae $B$ and hence in particular $v(B) \geq t$ for all $B \in T$, and $v(A)=[A]_{T}<[t]_{T}$ as required. We proceed by induction on the complexity of $B$, the atomic case holding by definition and the case of propositional connectives being easy. The quantifier cases follow using Lemma 2. 
Combining Lemmas 6 and 7 with Lemma 3 we obtain the following result:

Theorem 4. Let $\mathbf{L}$ be a core $\mathbf{U L}$-expansion and $T$ a countable theory. The following are equivalent:

(1) $T \vdash_{\forall \mathbf{L}^{\mathrm{D}}} A$.

(2) For every dense $L$-chain and safe valuation $v$ with non-empty domain $\mathcal{D}$, if $v(B) \geq$ $t$ for all $B \in T$, then $v(A) \geq t$.

In particular, $\vdash_{\forall \mathbf{L}}$ A iff $A$ is valid in all dense L-chains.

As an interesting aside, note that $C=\forall x(A(x) \odot B) \rightarrow(\forall x A(x) \odot B)$ is valid in all dense BL-chains [8], where BL is Hájek's Basic fuzzy logic, the logic of continuous $t$-norms. So by the preceding theorem $C$ is derivable in $\forall \mathbf{B L}^{\mathbf{D}}$. However, $C$ is not valid in all BL-chains [7] and hence not derivable in $\forall \mathbf{B L}$. The density rule is therefore not admissible for $\forall \mathbf{B L}$, and it remains an intriguing question as to whether $\forall \mathbf{B L}^{\mathbf{D}}$ can be obtained as an axiomatic extension of this logic.

\section{Rational Completeness}

We are ready now to put together the various pieces and establish rational completeness for a wide class of first-order logics; i.e. show completeness with respect to dense chains. First, we need a way of connecting axiomatizations with hypersequent calculi.

Definition 13. The standard interpretation function $I$ is defined as follows:

1. $I(\Gamma \Rightarrow C)=\odot \Gamma \rightarrow C, I(\Rightarrow C)=C, I(\Gamma \Rightarrow)=\odot \Gamma \rightarrow \perp$, and $I(\Rightarrow)=\perp$.

2. $I\left(\Gamma_{1} \Rightarrow \Delta_{1}|\ldots| \Gamma_{n} \Rightarrow \Delta_{n}\right)=I\left(\Gamma_{1} \Rightarrow \Delta_{1}\right) \vee \ldots \vee I\left(\Gamma_{n} \Rightarrow \Delta_{n}\right)$.

Lemma 8. Let $\mathbf{L}$ be a core $\mathbf{U L}$-expansion and $\mathbf{L}_{\mathbf{L}}$ a (first-order) w-simple sequent calculus whose rules are reductive and substitutive, such that $\vdash_{\mathbf{H L}_{\mathbf{L}}^{\mathbf{C}}} G$ iff $\vdash_{\forall \mathbf{L}} I(G)$ :

$$
\text { (a) } \vdash_{\mathbf{H L}_{\mathbf{L}}^{\mathbf{C}}+(D)} G \text { iff } \vdash_{\forall \mathbf{L}^{\mathrm{D}}} I(G) . \quad \text { (b) } \vdash_{\forall \mathbf{L}} A \text { iff } \vdash_{\forall \mathbf{L}^{\mathrm{D}}} A .
$$

Proof. (a) For the left-to-right direction, suppose that $\vdash_{\forall \mathbf{L}} I(G) \vee I\left(\Gamma_{1}, p \Rightarrow \Delta\right) \vee$ $I\left(\Gamma_{2} \Rightarrow p\right)$ where $p$ does not occur in $G, \Gamma_{1}, \Gamma_{2}$, or $\Delta$. It follows easily that $\vdash_{\forall \mathbf{L}} I(G) \vee$ $\left(p \rightarrow I\left(\Gamma_{1} \Rightarrow \Delta\right)\right) \vee I\left(\Gamma_{2} \Rightarrow p\right)$, and hence by (density), that $\vdash_{\forall \mathbf{L}^{\mathrm{D}}} I(G) \vee I\left(\Gamma_{1}, \Gamma_{2} \Rightarrow\right.$ $\Delta)$ as required. For the right-to-left direction, it is sufficient to show that (density) (with $T=\emptyset$ ) is admissible for $\mathbf{H L}_{\mathbf{L}}^{\mathbf{C}}+(D)$. If $\vdash_{\mathbf{H L}_{\mathbf{L}}^{\mathbf{C}}+(D)} \Rightarrow(A \rightarrow p) \vee(p \rightarrow B) \vee C$ where $p$ does not occur in $A, B$, or $C$, then (easily) $\vdash_{\mathbf{H L}_{\mathrm{L}}^{\mathbf{C}}+(D)} A \Rightarrow p|p \Rightarrow B| \Rightarrow C$. Hence by $(D), \vdash_{\mathbf{H L}_{\mathrm{L}}^{\mathrm{C}}+(D)} A \Rightarrow B \mid \Rightarrow C$. Using $(E C),(\vee, r)_{1},(\vee, r)_{2}$, and $(\rightarrow, r)$, it follows that $\vdash_{\mathbf{H L}_{\mathrm{L}}^{\mathrm{C}}+(D)} \Rightarrow(A \rightarrow B) \vee C$ as required.

(b) The left-to-right direction is trivial. For the right-to-left direction, suppose that $\vdash_{\forall \mathbf{L}^{\mathbf{D}}} A$. Then by (a), $\vdash_{\mathbf{H L}_{\mathbf{L}}^{\mathbf{C}}+(D)} \Rightarrow A$, and by Theorem $2, \vdash_{\mathbf{H L}_{\mathbf{L}}^{\mathbf{C}}} \Rightarrow A$. Hence by hypothesis and Definition $13, \vdash \forall \mathbf{L} A$.

Our main theorem now states that the first order version of any core UL-expansion which has a suitable hypersequent calculus is rational complete. 
Theorem 5. Let $\mathbf{L}$ be a core $\mathbf{U L}$-expansion and $\mathbf{L}_{\mathbf{L}}$ a (first-order) w-simple sequent calculus whose rules are reductive and substitutive, such that $\vdash_{\mathbf{H L}_{\mathbf{L}}^{\mathbf{C}}} G$ iff $\vdash_{\forall \mathbf{L}} I(G)$. Then $\forall \mathbf{L}$ is rational complete, i.e. the following are equivalent:

(1) $T \vdash_{\forall \mathbf{L}} A$.

(2) For every dense $L$-chain and safe valuation $v$ with non-empty domain $\mathcal{D}$, if $v(B) \geq$ $t$ for all $B \in T$, then $v(A) \geq t$.

In particular, $\vdash_{\forall \mathbf{L}} A$ iff $A$ is valid in all dense L-chains.

Proof. From (1) to (2) is easy. If (2) holds, then by Theorem $4, T \vdash_{\forall \mathbf{L}^{\mathrm{D}}} A$. By Lemma 5 (a), there is a finite subset $T_{0}$ of $T$ such that $T_{0} \vdash_{\forall \mathbf{L}^{\mathrm{D}}} A$, and by Lemma 5 (b), $\vdash_{\forall \mathbf{L}^{\mathrm{D}}} C \rightarrow A$ for some confusion $C$ of $T_{0}$. By Lemma $8(\mathrm{~b}), \vdash_{\forall \mathbf{L}} C \rightarrow A$ and, by Lemma 5 (c), $T_{0} \vdash_{\forall \mathbf{L}} C$. Hence, by $(m p), T_{0} \vdash_{\forall \mathbf{L}} A$ and therefore also $T \vdash_{\forall \mathbf{L}} A$.

For example, let $\forall$ SMTL and $\forall$ CnMTL $(n \geq 2)$ be the first-order versions of the logics SMTL [6] and CnMTL ( $n \geq 2)$ [4]. Hypersequent calculi for these logics, HL $_{\text {SMTL }}^{\mathrm{C}}$ and $\mathbf{H L}_{\text {CnMTL }}^{\mathrm{C}}$ are obtained by adding hypersequent versions of the rules $(w c)$ and $(n c)$ to the calculus $\mathbf{H} \forall \mathbf{F} \mathbf{L}_{\mathbf{e w}}^{\mathbf{C}}$ for $\forall \mathbf{M T L}$ (see Examples 1 and 3). Hence:

Corollary 1. $\forall$ MTL,$\forall$ SMTL, and $\forall$ CnMTL $(n \geq 2)$ are rational complete.

\section{References}

1. A. Avron. A constructive analysis of RM. Journal of Symbolic Logic, 52(4):939-951, 1987.

2. M. Baaz and R. Zach. Hypersequents and the proof theory of intuitionistic fuzzy logic. In Proceedings of CSL 2000, pages 187-201. LNCS, Springer-Verlag, 2000.

3. A. Ciabattoni. Automated generation of analytic calculi for logics with linearity. CSLO4: Computer Science Logic. LNCS, 3210:503-517, 2004.

4. A. Ciabattoni, F. Esteva, and L. Godo. T-norm based logics with $n$-contraction. Neural Network World, 12(5):441-453, 2002.

5. P. Cintula and P. Hájek. On theories and models in fuzzy predicate logics. Journal of Symbolic Logic, 71(3):832-863, 2006.

6. F. Esteva, J. Gispert, L. Godo, and F. Montagna. On the standard and rational completeness of some axiomatic extensions of the monoidal t-norm logic. Studia Logica, 71(2):199-226, 2002.

7. F. Esteva and L. Godo. Monoidal t-norm based logic: towards a logic for left-continuous t-norms. Fuzzy Sets and Systems, 124:271-288, 2001.

8. P. Hájek. Metamathematics of Fuzzy Logic. Kluwer, Dordrecht, 1998.

9. S. Jenei and F. Montagna. A proof of standard completeness for Esteva and Godo's MTL logic. Studia Logica, 70(2):183-192, 2002.

10. G. Metcalfe and F. Montagna. Substructural fuzzy logics. To appear in Journal of Symbolic Logic. http://www.math.vanderbilt.edu/people/metcalfe/publications.

11. F. Montagna and H. Ono. Kripke semantics, undecidability and standard completeness for Esteva and Godo's logic MTL $\forall$. Studia Logica, 71(2):227-245, 2002.

12. H. Ono and Y. Komori. Logic without the contraction rule. Journal of Symbolic Logic, 50:169-201, 1985.

13. M. Takano. Another proof of the strong completeness of the intuitionistic fuzzy logic. Tsukuba J. Math., 11:851-866, 1984.

14. G. Takeuti and T. Titani. Intuitionistic fuzzy logic and intuitionistic fuzzy set theory. Journal of Symbolic Logic, 49(3):851-866, 1984. 